\title{
On Differential Equations Derived from the Pseudospherical Surfaces
}

\author{
Hongwei Yang, ${ }^{1}$ Xiangrong Wang, ${ }^{1}$ and Baoshu Yin ${ }^{2,3}$ \\ ${ }^{1}$ College of Mathematics and Systems Science, Shandong University of Science and Technology, Qingdao 266590, China \\ ${ }^{2}$ Institute of Oceanology, Chinese Academy of Sciences, Qingdao 266071, China \\ ${ }^{3}$ Key Laboratory of Ocean Circulation and Wave, Chinese Academy of Sciences, Qingdao 266071, China
}

Correspondence should be addressed to Baoshu Yin; baoshuyin@126.com

Received 2 January 2014; Revised 19 March 2014; Accepted 21 March 2014; Published 24 April 2014

Academic Editor: Weiguo Rui

Copyright (C) 2014 Hongwei Yang et al. This is an open access article distributed under the Creative Commons Attribution License, which permits unrestricted use, distribution, and reproduction in any medium, provided the original work is properly cited.

\begin{abstract}
We construct two metric tensor fields; by means of these metric tensor fields, sinh-Gordon equation and elliptic sinh-Gordon equation are obtained, which describe pseudospherical surfaces of constant negative Riemann curvature scalar $\sigma=-2, \sigma=-1$, respectively. By employing the Bäcklund transformation, nonlinear superposition formulas of sinh-Gordon equation and elliptic sinh-Gordon equation are derived; various new exact solutions of the equations are obtained.
\end{abstract}

\section{Introduction}

The soliton equation [1] is related to several fields in mathematics [2] (such as differential geometry and nonlinear partial differential equation $[3,4]$ ) and theoretical physics (such as Josephson transition line [5], solitary Rossby waves and internal solitary waves in the ocean [6-9], chain of coupled pendula [10], pulse propagation in two-level atomic system [11], and quantum field theory [12]). Soliton equation can be derived from pseudospherical surfaces. Extensions to other soliton equations are straightforward. Soliton equations have several remarkable properties in common. Firstly, the initial value problem can be solved exactly by means of the inverse scattering methods [13]. Secondly, they have an infinite number of conservation laws $[14,15]$. Thirdly, they have Bäcklund transformations [16, 17]. Fourthly, they pass the Painlevé test [18]. Furthermore they describe pseudospherical surfaces, that is, surfaces of constant negative Gaussian curvature $[19,20]$.

Sinh-Gordon equation and elliptic sinh-Gordon equation are two important soliton equations in the field of soliton. From the model building perspective, there are various interesting examples making use of the sinh-Gordon equation and elliptic sinh-Gordon equation [21], such as the propagation of splay waves on a lipid membrane, one-dimensional models for elementary particles, selfinduced transparency of short optical pulses, and domain walls in ferroelectric and ferromagnetic materials. The second point worth noting is the historical development of the equations. They first appeared in differential geometry, where they were used to describe surfaces with a constant negative Gaussian curvature, but the previous study mainly focuses on sine-Gordon equation [22-25]; there are few scholarstic research on sinh-Gordon equation and elliptic sinh-Gordon equation.

In this paper, we will first construct two metric tensor fields; through these metric tensor fields, sinh-Gordon equations and elliptic sinh-Gordon equation are obtained. The method to derive soliton equations is greatly different from the previous papers [26]. Then, we will discuss analytic solutions of the sinh-Gordon equation and elliptic sinh-Gordon equation by using Bäcklund transformation. On the basis of the Bäcklund transformation, the formulas of nonlinear superposition of sinh-Gordon equation and elliptic sinhGordon equation are proposed in this paper, and the singlesoliton (breather) solution and double-soliton (breather) solution have been calculated. Finally, computer simulations of the single-soliton (breather) solution and double-soliton (breather) solution are presented by using the mathematical software Matlab. 


\section{General Method to Derive Soliton Equations from Pseudospherical Surfaces}

Metric tensor is used to study the invariant quantity of a surface [27, 28], such as the length of a curve drawn along the surface, the angle between a pair of curves drawn along the surface, and meeting at a common point, or tangent vectors at the same point of the surface, the area of a piece of the surface, and so on. However, many PDEs describe constant curvature surfaces. So, we can derive PDE via metric tensor. In this section, we introduce the general procedure for deriving soliton equations from pseudospherical surfaces. The metric tensor field for the PDE is given by

$$
f=f_{11} d x \otimes d x+f_{12} d x \otimes d t+f_{21} d t \otimes d x+f_{22} d t \otimes d t,
$$

and the line element is

$$
\left(\frac{d s}{d \lambda}\right)^{2}=f_{11}\left(\frac{d x}{d \lambda}\right)^{2}+\left(f_{12}+f_{21}\right) \frac{d x}{d \lambda} \frac{d t}{d \lambda}+f_{22}\left(\frac{d t}{d \lambda}\right)^{2} .
$$

The quantity $f$ can be written in matrix form

$$
f=\left(\begin{array}{ll}
f_{11} & f_{12} \\
f_{21} & f_{22}
\end{array}\right),
$$

and then the inverse of $f$ is given by

$$
f^{-1}=\left(\begin{array}{ll}
f^{11} & f^{12} \\
f^{21} & f^{22}
\end{array}\right) \text {. }
$$

Next we have to calculate the Christoffel symbols. They are defined as

$$
\tau_{m n}^{a}:=\sum_{b} \frac{1}{2} f^{a b}\left(f_{b m, n}+f_{b n, m}-f_{m n, b}\right)
$$

where

$$
f_{b m, 1}:=\frac{\partial f_{b m}}{\partial x}, \quad f_{b m, 2}=\frac{\partial f_{b m}}{\partial t} .
$$

Following, we calculate the Riemann curvature tensor which is given by

$$
\sigma_{m s q}^{r}:=\tau_{m q, s}^{r}-\tau_{m s, q}^{r}+\sum_{n}\left(\tau_{n s}^{r} \tau_{m q}^{n}-\tau_{n q}^{r} \tau_{m s}^{n}\right) .
$$

The Ricci tensor follows as

$$
\sigma_{m q}:=\sigma_{m a q}^{a}=-\sigma_{m q a}^{a},
$$

and is constructed by contraction. From $\sigma_{n q}$, we obtain $\sigma_{q}^{m}$ via

$$
\sigma_{q}^{m}=f^{m n} \sigma_{n q}
$$

Finally, the curvature scalar $\sigma$ is given by

$$
\sigma:=\sigma_{m}^{m}
$$

If the given $\sigma$ is a constant, we will get a partial differential equation.
2.1. Sinh-Gordon Equation Derived from Pseudospherical Surfaces. Sinh-Gordon equation and elliptic sinh-Gordon equation appear in wide range of physical applications including integrable quantum field theory, kink dynamics, fluid dynamics, and nonlinear optics [29-31]. In this section, we will derive sinh-Gordon equation from pseudospherical surfaces following the method presented in the previous section. The metric tensor field for the sinh-Gordon equation is given by

$$
\begin{aligned}
f= & d x \otimes d x+\cosh (u(x, t)) d x \otimes d t \\
& +\cosh (u(x, t)) d t \otimes d x+d t \otimes d t,
\end{aligned}
$$

and the line element is

$$
\left(\frac{d s}{d \lambda}\right)^{2}=\left(\frac{d x}{d \lambda}\right)^{2}+2 \cosh (u(x, t)) \frac{d x}{d \lambda} \frac{d t}{d \lambda}+\left(\frac{d t}{d \lambda}\right)^{2}
$$

where $u$ is a smooth function of $x$ and $t$. Firstly, we will calculate the Riemann curvature scalar $\sigma$ from $f$. Then the sinh-Gordon equation follows when we impose the condition $\sigma=-2$. We have

$$
f_{11}=f_{22}=1, \quad f_{21}=f_{12}=\cosh (u) .
$$

The quantity $f$ can be written in matrix form

$$
f=\left(\begin{array}{ll}
f_{11} & f_{12} \\
f_{21} & f_{22}
\end{array}\right)
$$

and the inverse of $f$ is given by

$$
f^{-1}=\left(\begin{array}{ll}
f^{11} & f^{12} \\
f^{21} & f^{22}
\end{array}\right)
$$

where

$$
f^{11}=f^{22}=-\frac{1}{\sinh ^{2}(u)}, \quad f^{12}=f^{21}=\frac{\cosh (u)}{\sinh ^{2}(u)} .
$$

Differentiating (14) with respect to $x$ and $t$, we obtain

$$
f_{x}=\left(\begin{array}{ll}
f_{11,1} & f_{12,1} \\
f_{21,1} & f_{22,1}
\end{array}\right), \quad f_{t}=\left(\begin{array}{ll}
f_{11,2} & f_{12,2} \\
f_{21,2} & f_{22,2}
\end{array}\right),
$$

where

$$
\begin{gathered}
f_{x}=\frac{\partial f}{\partial x}, \quad f_{11,1}=f_{22,1}=0 \\
f_{12,1}=f_{21,1}=\sinh (u) u_{x} \\
f_{t}=\frac{\partial f}{\partial t}, \quad f_{11,2}=f_{22,2}=0 \\
f_{12,2}=f_{21,2}=\sinh (u) u_{t} .
\end{gathered}
$$

Since

$$
\tau_{m n}^{a}=\frac{1}{2} f^{a b}\left(f_{b m, n}+f_{b n, m}-f_{m n, b}\right)
$$


we obtain

$$
\begin{array}{cl}
\tau_{11}^{1}=\frac{\cosh (u) u_{x}}{\sinh (u)}, & \tau_{11}^{2}=-\frac{u_{x}}{\sinh (u)}, \\
\tau_{12}^{1}=0, \quad \tau_{12}^{2}=0, & \tau_{21}^{1}=0, \quad \tau_{21}^{2}=0, \\
\tau_{22}^{1}=-\frac{u_{t}}{\sinh (u)}, & \tau_{22}^{2}=\frac{\cosh (u) u_{t}}{\sinh (u)} .
\end{array}
$$

Differentiating (20) with respect to $x$ and $t$, we obtain

$$
\begin{aligned}
& \tau_{11,1}^{1}=\frac{\cosh (u) \sinh (u) u_{x x}-\left(u_{x}\right)^{2}}{\sinh ^{2}(u)}, \\
& \tau_{11,2}^{1}= \frac{\cosh (u) \sinh ^{2}(u) u_{x t}-u_{x} u_{t}}{\sinh ^{2}(u)}, \\
& \tau_{11,1}^{2}= \frac{\cosh (u)\left(u_{x}\right)^{2}-\sinh (u) u_{x x}}{\sinh ^{2}(u)}, \\
& \tau_{11,2}^{2}= \frac{\cosh (u) u_{t} u_{x}-\sinh (u) u_{x t}}{\sinh ^{2}(u)}, \\
& \tau_{12,1}^{1}=\tau_{12,2}^{1}=\tau_{12,1}^{2}=\tau_{12,2}^{2}=\tau_{21,1}^{1} \\
&=\tau_{21,2}^{1}=\tau_{21,1}^{2}=\tau_{21,2}^{2}=0, \\
& \tau_{22,1}^{1}=\frac{\cosh (u) u_{x} u_{t}-\sinh (u) u_{x t}}{\sinh ^{2}(u)}, \\
& \tau_{22,2}^{1}=\frac{\cosh (u)\left(u_{t}\right)^{2}-\sinh (u) u_{t t}}{\sinh ^{2}(u)} \\
& \tau_{22,1}^{2}=\frac{\cosh (u) \sinh ^{2}(u) u_{x t}-u_{x} u_{t}}{\sinh ^{2}(u)}, \\
& \tau_{22,2}^{2}=\frac{\cosh (u) \sinh ^{2}(u) u_{t t}-\left(u_{t}\right)^{2}}{\sinh ^{2}(u)} .
\end{aligned}
$$

By virtue of

$$
\sigma_{m s q}^{r}:=\tau_{m q, s}^{r}-\tau_{m s, q}^{r}+\sum_{n}\left(\tau_{n s}^{r} \tau_{m q}^{n}-\tau_{n q}^{r} \tau_{m s}^{n}\right)
$$

we get

$$
\begin{gathered}
\sigma_{111}^{1}=0, \quad \sigma_{112}^{1}=-\frac{\cosh (u) u_{x t}}{\sinh (u)}, \\
\sigma_{211}^{1}=0, \quad \sigma_{212}^{1}=-\frac{u_{x t}}{\sinh (u)} \\
\sigma_{121}^{2}=-\frac{u_{x t}}{\sinh (u)}, \quad \sigma_{122}^{2}=0, \\
\sigma_{221}^{2}=-\frac{\cosh (u) u_{x t}}{\sinh (u)}, \quad \sigma_{222}^{2}=0 .
\end{gathered}
$$

By virtue of

$$
\sigma_{m q}:=\sigma_{m a q}^{a}=-\sigma_{m q a}^{a}
$$

we get

$$
\begin{aligned}
& \sigma_{11}=-\frac{u_{x t}}{\sinh (u)}, \quad \sigma_{12}=-\frac{\cosh (u) u_{x t}}{\sinh (u)} \\
& \sigma_{21}=-\frac{\cosh (u) u_{x t}}{\sinh (u)}, \quad \sigma_{22}=-\frac{u_{x t}}{\sinh (u)} .
\end{aligned}
$$

By virtue of

$$
\sigma_{q}^{m}=f^{m n} \sigma_{n q},
$$

we get

$$
\sigma_{1}^{1}=-\frac{u_{x t}}{\sinh (u)}, \quad \sigma_{2}^{2}=-\frac{u_{x t}}{\sinh (u)} .
$$

Finally, with the help of

$$
\sigma:=\sigma_{m}^{m}
$$

we get

$$
\sigma:=-\frac{2 u_{x t}}{\sinh (u)} .
$$

When given $\sigma=-2$, the well-known sinh-Gordon equation

$$
u_{x t}=\sinh (u)
$$

is obtained.

2.2. Elliptic Sinh-Gordon Equation Derived from Pseudospherical Surfaces. In this section, we will derive elliptic sinhGordon equation from pseudospherical surfaces. The metric tensor field for the elliptic sinh-Gordon equation is given by

$$
\begin{aligned}
f= & \cosh (u(x, t)) d x \otimes d x+d x \otimes d t \\
& +d t \otimes d x+\cosh (u(x, t)) d t \otimes d t,
\end{aligned}
$$

and the line element is

$$
\begin{aligned}
\left(\frac{d s}{d \lambda}\right)^{2}= & \cosh (u(x, t))\left(\frac{d x}{d \lambda}\right)^{2}+2 \frac{d x}{d \lambda} \frac{d t}{d \lambda} \\
& +\cosh (u(x, t))\left(\frac{d t}{d \lambda}\right)^{2} .
\end{aligned}
$$

Firstly, we calculate the Riemann curvature scalar $\sigma$ from $f$. Then the elliptic sinh-Gordon equation follows when we impose the condition $\sigma=-1$. We have

$$
f_{11}=f_{22}=\cosh (u), \quad f_{21}=f_{12}=1 .
$$

The quantity $f$ can be written in matrix form

$$
f=\left(\begin{array}{ll}
f_{11} & f_{12} \\
f_{21} & f_{22}
\end{array}\right),
$$


and the inverse of $f$ is given by

$$
f^{-1}=\left(\begin{array}{ll}
f^{11} & f^{12} \\
f^{21} & f^{22}
\end{array}\right)
$$

where

$$
f^{11}=f^{22}=\frac{\cosh (u)}{\sinh ^{2}(u)}, \quad f^{12}=f^{21}=-\frac{1}{\sinh ^{2}(u)} .
$$

Differentiate (34) with respect to $x$ and $t$, we have

$$
f_{x}=\left(\begin{array}{ll}
f_{11,1} & f_{12,1} \\
f_{21,1} & f_{22,1}
\end{array}\right), \quad f_{t}=\left(\begin{array}{ll}
f_{11,2} & f_{12,2} \\
f_{21,2} & f_{22,2}
\end{array}\right),
$$

where

$$
\begin{gathered}
f_{x}=\frac{\partial f}{\partial x}, \quad f_{11,1}=f_{22,1}=\sinh (u) u_{x} \\
f_{12,1}=f_{21,1}=0 \\
f_{t}=\frac{\partial f}{\partial t}, \quad f_{11,2}=f_{22,2}=\sinh (u) u_{t}, \\
f_{12,1}=f_{21,1}=0 .
\end{gathered}
$$

Since

$$
\tau_{m n}^{a}=\frac{1}{2} f^{a b}\left(f_{b m, n}+f_{b n, m}-f_{m n, b}\right)
$$

we obtain

$$
\begin{array}{cc}
\tau_{11}^{1}=\frac{\cosh (u) u_{x}+u_{t}}{2 \sinh (u)}, & \tau_{11}^{2}=-\frac{u_{x}+\cosh (u) u_{t}}{2 \sinh (u)}, \\
\tau_{12}^{1}=\frac{\cosh (u) u_{t}-u_{x}}{2 \sinh (u)}, & \tau_{12}^{2}=\frac{-u_{t}+\cosh (u) u_{x}}{2 \sinh (u)}, \\
\tau_{21}^{1}=\frac{\cosh (u) u_{t}-u_{x}}{2 \sinh (u)}, & \tau_{21}^{2}=\frac{-u_{t}+\cosh (u) u_{x}}{2 \sinh (u)}, \\
\tau_{22}^{1}=-\frac{\cosh (u) u_{x}+u_{t}}{2 \sinh (u)}, & \tau_{22}^{2}=\frac{u_{x}+\cosh (u) u_{t}}{2 \sinh (u)} .
\end{array}
$$

Differentiating (40) with respect to $x$ and $t$, we obtain

$$
\begin{gathered}
\tau_{11,1}^{1}=\frac{-\cosh (u) u_{x} u_{t}+\cosh (u) \sinh (u) u_{x x}+\sinh (u) u_{x t}-\left(u_{x}\right)^{2}}{2 \sinh ^{2}(u)}, \\
\tau_{11,2}^{1}=\frac{-\cosh (u)\left(u_{t}\right)^{2}+\cosh (u) \sinh (u) u_{x t}+\sinh (u) u_{t t}-u_{x} u_{t}}{2 \sinh ^{2}(u)},
\end{gathered}
$$

$$
\begin{aligned}
& \tau_{11,1}^{2}=-\frac{\cosh (u) \sinh (u) u_{x t}-\cosh (u)\left(u_{x}\right)^{2}-u_{x} u_{t}+\sinh (u) u_{x x}}{2 \sinh ^{2}(u)}, \\
& \tau_{11,2}^{2}=-\frac{\cosh (u) \sinh (u) u_{t t}-\cosh (u) u_{x} u_{t}-\left(u_{t}\right)^{2}+\sinh (u) u_{x t}}{2 \sinh ^{2}(u)}, \\
& \tau_{12,1}^{1}=\frac{\cosh (u)\left(u_{x}\right)^{2}+\cosh (u) \sinh (u) u_{x t}-u_{x} u_{t}-\sinh (u) u_{x x}}{2 \sinh ^{2}(u)}, \\
& \tau_{12,1}^{2}=\frac{\cosh (u) u_{x} u_{t}+\cosh (u) \sinh (u) u_{t t}-\left(u_{t}\right)^{2}-\sinh (u) u_{x t}}{2 \sinh ^{2}(u)}, \\
& \tau_{12,2}^{1}=\frac{\cosh (u) u_{x} u_{t}+\cosh (u) \sinh (u) u_{x x}-\left(u_{x}\right)^{2}-\sinh (u) u_{x t}}{2 \sinh ^{2}(u)}, \\
& \tau_{12,2}^{2}=\frac{\cosh (u)\left(u_{t}\right)^{2}+\cosh (u) \sinh (u) u_{x t}-u_{x} u_{t}-\sinh (u) u_{t t}}{2 \sinh ^{2}(u)}, \\
& \tau_{21,1}^{1}=\frac{\cosh (u)\left(u_{x}\right)^{2}+\cosh (u) \sinh (u) u_{x t}-u_{x} u_{t}-\sinh (u) u_{x x}}{2 \sinh ^{2}(u)} \\
& \tau_{21,2}^{1}=\frac{\cosh (u) u_{x} u_{t}+\cosh (u) \sinh (u) u_{t t}-\left(u_{t}\right)^{2}-\sinh (u) u_{x t}}{2 \sinh ^{2}(u)}, \\
& \tau_{21,1}^{2}=\frac{\cosh (u) u_{x} u_{t}+\cosh (u) \sinh (u) u_{x x}-\left(u_{x}\right)^{2}-\sinh (u) u_{x t}}{2 \sinh ^{2}(u)}, \\
& \tau_{21,2}^{2}=\frac{\cosh (u)\left(u_{t}\right)^{2}+\cosh (u) \sinh (u) u_{x t}-u_{x} u_{t}-\sinh (u) u_{t t}}{2 \sinh ^{2}(u)}, \\
& \tau_{22,1}^{1}=\frac{\cosh (u) u_{x} u_{t}-\cosh (u) \sinh (u) u_{x x}-\sinh (u) u_{x t}+\left(u_{x}\right)^{2}}{2 \sinh ^{2}(u)},
\end{aligned}
$$$$
\tau_{22,2}^{1}=\frac{\cosh (u)\left(u_{t}\right)^{2}-\cosh (u) \sinh (u) u_{x t}-\sinh (u) u_{t t}+u_{x} u_{t}}{2 \sinh ^{2}(u)},
$$$$
\tau_{22,1}^{2}=\frac{\cosh (u) \sinh (u) u_{x t}-\cosh (u)\left(u_{x}\right)^{2}-u_{x} u_{t}+\sinh (u) u_{x x}}{2 \sinh ^{2}(u)},
$$$$
\tau_{22,2}^{2}=\frac{\cosh (u) \sinh (u) u_{t t}-\cosh (u) u_{x} u_{t}-\left(u_{t}\right)^{2}+\sinh (u) u_{x t}}{2 \sinh ^{2}(u)} .
$$

By virtue of

$$
\sigma_{m s q}^{r}:=\tau_{m q, s}^{r}-\tau_{m s, q}^{r}+\sum_{n}\left(\tau_{n s}^{r} \tau_{m q}^{n}-\tau_{n q}^{r} \tau_{m s}^{n}\right)
$$

we get

$$
\begin{gathered}
\sigma_{111}^{1}=0, \quad \sigma_{112}^{1}=-\frac{u_{x x}+u_{t t}}{2 \sinh (u)}, \\
\sigma_{211}^{1}=0, \quad \sigma_{212}^{1}=-\frac{\left(u_{t t}+u_{x x}\right) \cosh (u)}{2 \sinh (u)} \\
\sigma_{121}^{2}=-\frac{\left(u_{t t}+u_{x x}\right) \cosh (u)}{2 \sinh (u)}, \quad \sigma_{122}^{2}=0, \\
\sigma_{221}^{2}=-\frac{u_{t t}+u_{x x}}{2 \sinh (u)}, \quad \sigma_{222}^{2}=0 .
\end{gathered}
$$


By virtue of

$$
\sigma_{m q}:=\sigma_{m a q}^{a}=-\sigma_{m q a}^{a},
$$

we get

$$
\begin{gathered}
\sigma_{11}=-\frac{\left(u_{t t}+u_{x x}\right) \cosh (u)}{2 \sinh (u)}, \quad \sigma_{12}=-\frac{u_{t t}+u_{x x}}{2 \sinh (u)} \\
\sigma_{21}=-\frac{u_{t t}+u_{x x}}{2 \sinh (u)}, \quad \sigma_{22}=-\frac{\left(u_{t t}+u_{x x}\right) \cosh (u)}{2 \sinh (u)} .
\end{gathered}
$$

By virtue of

$$
\sigma_{q}^{m}=f^{m n} \sigma_{n q}
$$

we get

$$
\sigma_{1}^{1}=-\frac{u_{x x}+u_{t t}}{2 \sinh (u)}, \quad \sigma_{2}^{2}=-\frac{u_{x x}+u_{t t}}{2 \sinh (u)} .
$$

Finally, with the help of

$$
\sigma:=\sigma_{m}^{m}
$$

we get

$$
\sigma:=-\frac{u_{x x}+u_{t t}}{\sinh (u)}
$$

When given $\sigma=-1$, the well-known elliptic sinh-Gordon equation

$$
u_{x x}+u_{t t}=\sinh (u)
$$

is obtained.

\section{Solutions to the Sinh-Gordon Equation and Elliptic Equation}

Bäcklund transformations play an important role in finding solutions of a certain class of nonlinear partial differential equations $[32,33]$. From a solution of a nonlinear partial differential equation, we can sometimes find a relationship that will generate the solution of a different partial differential equation, which is known as a Bäcklund transformation, or of the same partial differential equation where such a relation is then known as an auto-Bäcklund transformation.

As to elliptic sinh-Gordon equation

$$
u_{x x}+u_{t t}=\sinh (u)
$$

under the transformation

$$
x \longmapsto \frac{b}{2}(x-i t), \quad t \longmapsto \frac{1}{2 b}(x+i t),
$$

where $b$ is a positive constant, (51) is transformed into the sinh-Gordon equation

$$
u_{x t}=\sinh (u)
$$

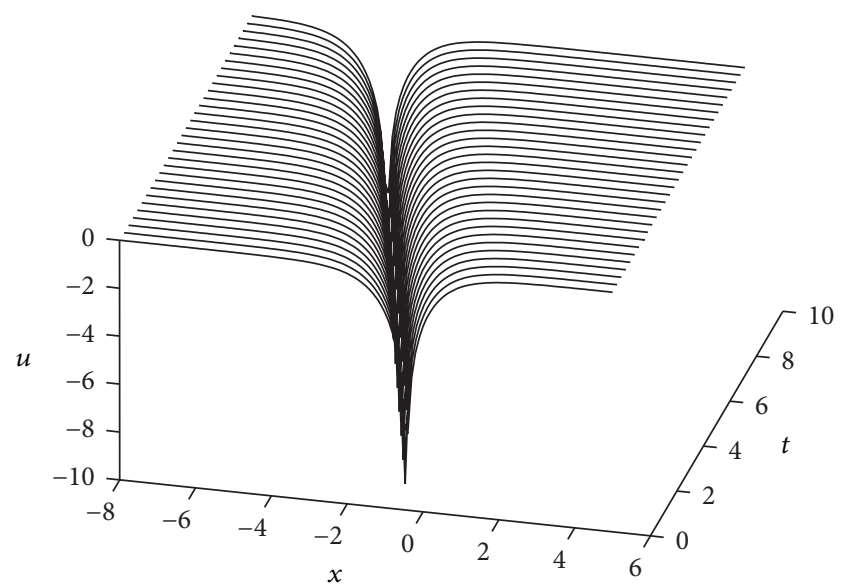

FIgure 1: Waterfall of (58), where $\alpha=1.5, C=0$.

So, if we get the solutions of the sinh-Gordon equation, it is very easy to get the solutions of the elliptic sinh-Gordon equation.

The auto-Bäcklund transformations for the sinh-Gordon equation

$$
u_{x t}=\sinh (u)
$$

is given by

$$
\begin{aligned}
& \left(\frac{u^{\prime}+u}{2}\right)_{x}=a \sinh \left(\frac{u^{\prime}-u}{2}\right), \\
& \left(\frac{u^{\prime}-u}{2}\right)_{t}=\frac{1}{a} \sinh \left(\frac{u^{\prime}+u}{2}\right) .
\end{aligned}
$$

If $u$ is a solution of the sinh-Gordon equation, $u^{\prime}$ is also a solution of the sinh-Gordon equation. Here we are looking for solutions of the sinh-Gordon equation by using the Bäcklund transformations. Obviously $u(x, t)=0$ is a solution of the sinh-Gordon equation. This is known as the vacuum solution. We make use of the auto-Bäcklund transformation to construct another solution of the sinh-Gordon equation from the vacuum solution. Inserting this solution into the given Bäcklund transformation results in

$$
\left(\frac{u^{\prime}}{2}\right)_{x}=a \sinh \left(\frac{u^{\prime}}{2}\right), \quad\left(\frac{u^{\prime}}{2}\right)_{t}=\frac{1}{a} \sinh \left(\frac{u^{\prime}}{2}\right) .
$$

Since

$$
\int \frac{d u}{\sinh (u / 2)}=-4 \tanh ^{-1} \exp \left(\frac{u}{2}\right)
$$

we obtain a new solution of the sinh-Gordon equation; namely,

$$
u^{\prime}=2 \ln \tanh \left(-\frac{a}{2} x-\frac{1}{2 a} t+C\right)
$$

where $C$ is a constant of integration, and the computer simulation of (58) is presented in Figures 1 and 2. 


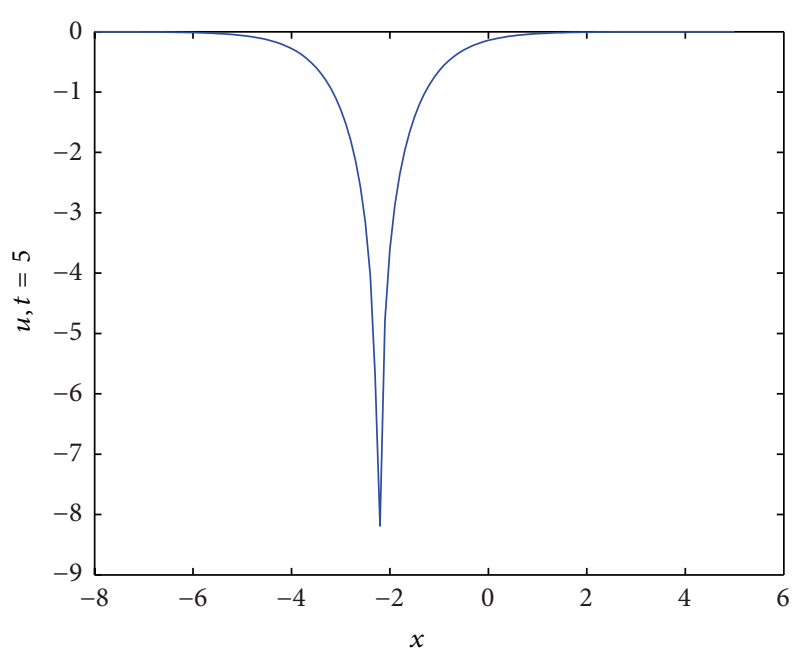

FIGURE 2: Solution of (58), where $\alpha=1.5, C=0$, and $t=5$.

This solution may be used to determine another solution for the sinh-Gordon equation and so on. If we use this method to calculate other new solutions, it is very difficult to solve the first-order equation. However, we can get the nonlinear superposition formula via (55). From $u_{0}$, first by employing $a_{1}, u_{1}$ is obtained; then by employing $a_{2}, u_{3}$ can be obtained:

$$
\begin{aligned}
& \left(\frac{u_{1}+u_{0}}{2}\right)_{x}=a_{1} \sinh \frac{u_{1}-u_{0}}{2}, \\
& \left(\frac{u_{3}+u_{1}}{2}\right)_{x}=a_{2} \sinh \frac{u_{3}-u_{1}}{2} .
\end{aligned}
$$

Meanwhile by changing the using order of $a_{1}$ and $a_{2}, u_{2}$ and $u_{4}$ are also obtained, respectively. If $u_{3}=u_{4}$, then

$$
\begin{aligned}
& \left(\frac{u_{2}+u_{0}}{2}\right)_{x}=a_{2} \sinh \frac{u_{2}-u_{0}}{2}, \\
& \left(\frac{u_{3}+u_{2}}{2}\right)_{x}=a_{1} \sinh \frac{u_{3}-u_{2}}{2} .
\end{aligned}
$$

From (59) and (60), we get

$$
\begin{aligned}
& a_{1}\left(\sinh \frac{u_{1}-u_{0}}{2}+\sinh \frac{u_{3}-u_{2}}{2}\right) \\
& \quad=a_{2}\left(\sinh \frac{u_{2}-u_{0}}{2}+\sinh \frac{u_{3}-u_{1}}{2}\right) .
\end{aligned}
$$

By simple calculation, (61) can be rewritten as

$$
\begin{aligned}
2 a_{1} & \sinh \frac{u_{1}-u_{0}+u_{3}-u_{2}}{4} \cosh \frac{u_{1}-u_{0}-u_{3}+u_{2}}{4} \\
& =2 a_{2} \sinh \frac{u_{2}-u_{0}+u_{3}-u_{1}}{4} \cosh \frac{u_{2}-u_{0}-u_{3}+u_{1}}{4},
\end{aligned}
$$

or

$$
\begin{gathered}
a_{1}\left(\sinh \frac{u_{3}-u_{0}}{4} \cosh \frac{u_{1}-u_{2}}{4}+\sinh \frac{u_{1}-u_{2}}{4} \cosh \frac{u_{3}-u_{0}}{4}\right) \\
=a_{2}\left(\sinh \frac{u_{3}-u_{0}}{4} \cosh \frac{u_{2}-u_{1}}{4}\right. \\
\left.+\sinh \frac{u_{2}-u_{1}}{4} \cosh \frac{u_{3}-u_{0}}{4}\right) .
\end{gathered}
$$

After abbreviation, the following nonlinear superposition formula obtained

$$
\tanh \frac{u_{3}-u_{0}}{4}=\frac{a_{2}+a_{1}}{a_{2}-a_{1}} \tanh \frac{u_{1}-u_{2}}{4}
$$

or

$$
u_{3}=u_{0}+4 \tanh ^{-1} \frac{a_{2}+a_{1}}{a_{2}-a_{1}} \tanh \frac{u_{1}-u_{2}}{4} .
$$

If we are given

$$
\begin{gathered}
u_{0}=0, \quad u_{1}=2 \ln \tanh \left(-\frac{a_{1}}{2} x-\frac{1}{2 a_{1}} t+C_{1}\right), \\
u_{2}=2 \ln \tanh \left(-\frac{a_{2}}{2} x-\frac{1}{2 a_{2}} t+C_{2}\right),
\end{gathered}
$$

by means of (65), we can easily get the fourth solution

$$
\begin{aligned}
u_{3}= & 4 \tanh ^{-1} \frac{a_{2}+a_{1}}{a_{2}-a_{1}} \\
\times \tanh ( & \left(\ln \tanh \left(-\frac{a_{1}}{2} x-\frac{1}{2 a_{1}} t+C_{1}\right)\right. \\
& \left.\left.\quad-\ln \tanh \left(-\frac{a_{2}}{2} x-\frac{1}{2 a_{2}} t+C_{2}\right)\right) \times(2)^{-1}\right),
\end{aligned}
$$

and the computer simulation of the solution is presented in Figures 3 and 4 .

In this way, by algebraic operation, a series of new solutions of sinh-Gordon equation can be easily obtained. Similarly, from (52), (58), and (67), we can get the single breather solution

$$
u^{\prime}=2 \ln \tanh \left(-\frac{1}{4}\left(\left(a b+\frac{1}{a b}\right) x-i\left(a b-\frac{1}{a b}\right) t\right)+C\right)
$$




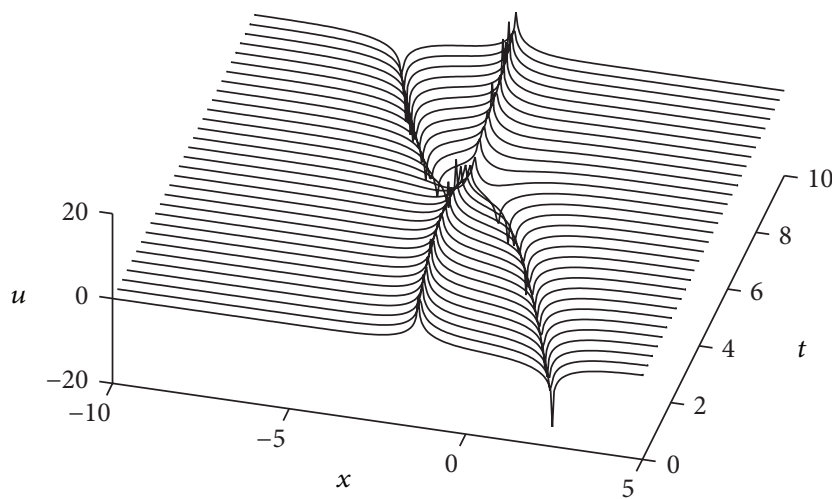

FIgURE 3: Waterfall of (67), where $C_{1}=1, C_{2}=0, \alpha_{1}=1.3$, and $\alpha_{2}=1.7$.

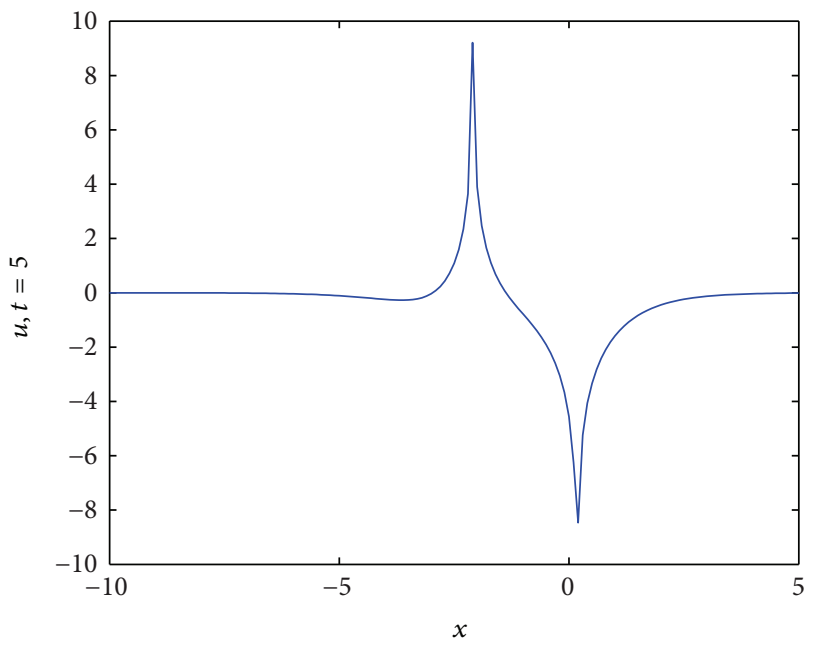

FIGURE 4: Solution of (67), where $C_{1}=1, C_{2}=0, \alpha_{1}=1.3, \alpha_{2}=1.7$, and $t=5$.

and double breather solution

$$
\begin{gathered}
u_{3}=4 \tanh ^{-1} \frac{a_{2}+a_{1}}{a_{2}-a_{1}} \\
\times \tanh \left(\operatorname { l n } \operatorname { t a n h } \left(-\frac{1}{4}\left(\left(a_{1} b+\frac{1}{a_{1} b}\right) x\right.\right.\right. \\
\left.\left.\quad-i\left(a_{1} b-\frac{1}{a_{1} b}\right) t\right)+C_{1}\right) \\
-\ln \tanh \left(-\frac{1}{4}\left(\left(a_{2} b+\frac{1}{a_{2} b}\right) x\right.\right. \\
\left.\quad \times(2)^{-1}\right)
\end{gathered}
$$

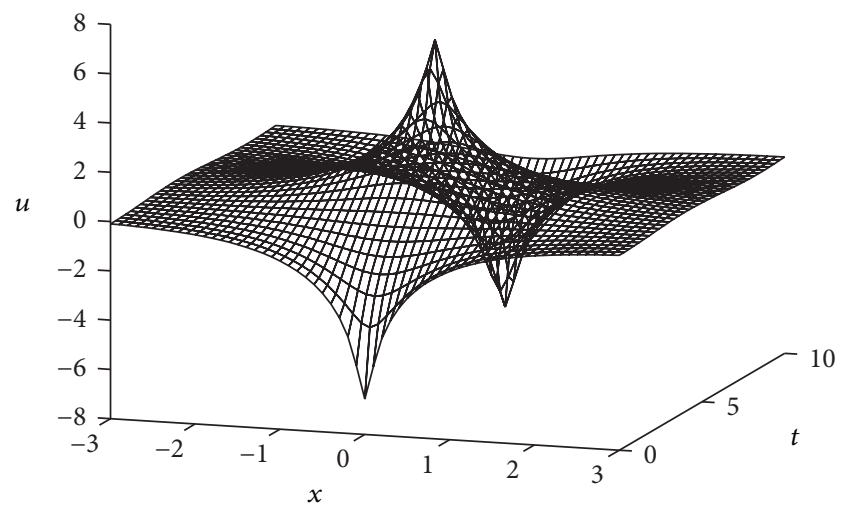

Figure 5: Waterfall of (68), where $\alpha=1, b=2$, and $C=0$.

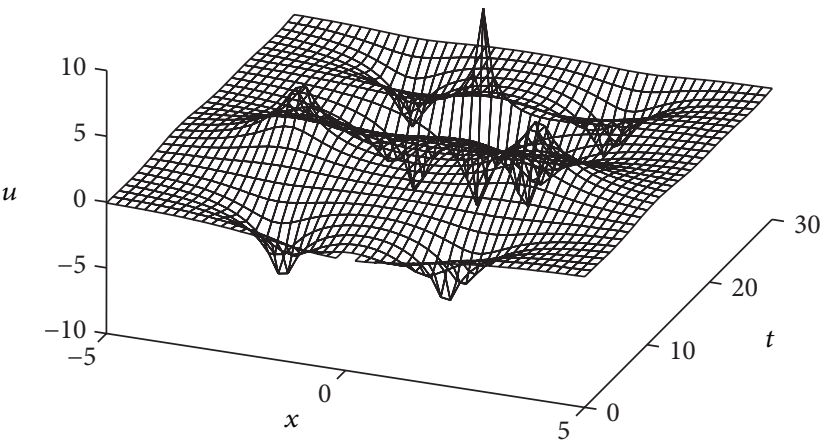

FIgURE 6: Waterfall of (69), where $C_{1}=0, C_{2}=0, \alpha_{1}=1.3, \alpha_{2}=1.7$, and $b=1$.

of the elliptic sinh-Gordon equation. The computer simulation of the solutions is presented in Figures 5 and 6.

\section{Summary and Discussion}

In this paper, we obtain sinh-Gordon equation and elliptic sinh-Gordon equation by means of pseudospherical surfaces. In addition, we give the Bäcklund transformations and nonlinear superposition formulas of sinh-Gordon equation and elliptic sinh-Gordon equation, which lead to new exact solutions of the sinh-Gordon equation and elliptic sinhGordon equation. On the basis of the Bäcklund transformations and nonlinear superposition formulas, the singlesoliton (breather) solution and double-soliton (breather) solution of the sinh-Gordon equation and elliptic sinhGordon equation have been calculated. Finally, computer simulations of the single-soliton (breather) solution and double-soliton (breather) solution are presented by using the mathematical software Matlab. In forthcoming days, we will further discuss the problem. It is also interesting for us to see how the metric tensor field will be for other soliton equations. 


\section{Conflict of Interests}

The authors declare that there is no conflict of interests regarding the publication of this paper.

\section{Acknowledgment}

This work was supported by the Strategic Pioneering Program of Chinese Academy of Sciences (no. XDA 10020104), the National Natural Science Foundation of China (no. 11271007), the Nature Science Foundation of Shandong Province of China (no. ZR2013AQ017), the Open Fund of the Key Laboratory of Data Analysis and Application, State Oceanic Administration (no. LDAA-2013-04), and the SDUST Research Fund (no. 2012KYTD105).

\section{References}

[1] E. Infeld and G. Rowlands, Nonlinear Waves, Solitons and Chaos, Cambridge University Press, Cambridge, UK, 2nd edition, 2000.

[2] A. Dold and B. Eckmann, Bäcklund Transformations, Lecture Note in Mathematics, Springer, New York, NY, USA, 1974.

[3] Y. F. Zhang and H. W. Tam, "Discussion on integrable properties for higher-dimensional variable-coefficient nonlinear partial differential equations," Journal of Mathematical Physics, vol. 54, Article ID 013516, 2013.

[4] T. C. Xia, H. Q. Zhang, and Z. Y. Yan, "New explicit and exact travelling wave solutions for a class of nonlinear evolution equations," Applied Mathematics and Mechanics, vol. 22, no. 7, pp. 788-793, 2001.

[5] A. C. Scott, "Propagation of magnetic flux on a long Josephson tunnel junction," Il Nuovo Cimento B Series 10, vol. 69, no. 2, pp. 241-261, 1970.

[6] H.-W. Yang, B.-S. Yin, D.-Z. Yang, and Z.-H. Xu, "Forced solitary Rossby waves under the influence of slowly varying topography with time," Chinese Physics B, vol. 20, no. 12, Article ID 120203, 2011.

[7] H. W. Yang, B. S. Yin, and Y. L. Shi, "Forced dissipative Boussinesq equation for solitary waves excited by unstable topography," Nonlinear Dynamics, vol. 70, no. 2, pp. 1389-1396, 2012.

[8] Z. H. Xu, B. S. Yin, Y. J. Hou et al., "Variability of internal tides and near-inertial waves on the continental slope of the northwestern South China Sea," Journal of Geophysical Research, vol. 118, pp. 197-211, 2013.

[9] Z. Xu, B. Yin, Y. Hou, Z. Fan, and A. K. Liu, "A study of internal solitary waves observed on the continental shelf in the northwestern South China Sea," Acta Oceanologica Sinica, vol. 29, no. 3, pp. 18-25, 2010.

[10] P. G. Drazin, Solitons, vol. 85 of London Mathematical Society Lecture Note Series, Cambridge University Press, London, UK, 1983.

[11] G. L. Lamb, Jr., "Analytical descriptions of ultrashort optical pulse propagation in a resonant medium," Reviews of Modern Physics, vol. 43, pp. 99-124, 1971.

[12] G. Feverati, F. Ravanini, and G. Takács, "Non-linear integral equation and finite volume spectrum of sine-Gordon theory," Nuclear Physics B, vol. 540, no. 3, pp. 543-586, 1999.
[13] M. J. Ablowitz and P. A. Clarkson, Solitons, Nonlinear Evolution Equations and Inverse Scattering, vol. 149 of London Mathematical Society Lecture Note Series, Cambridge University Press, New York, NY, USA, 1991.

[14] A. H. Khater, D. K. Callebaut, and S. M. Sayed, "Conservation laws for some nonlinear evolution equations which describe pseudo-spherical surfaces," Journal of Geometry and Physics, vol. 51, no. 3, pp. 332-352, 2004.

[15] W.-X. Ma, "Variational identities and applications to Hamiltonian structures of soliton equations," Nonlinear Analysis: Theory, Methods \& Applications, vol. 71, no. 12, pp. e1716-e1726, 2009.

[16] Y. Zhang, Z. Han, and H.-W. Tam, "An integrable hierarchy and Darboux transformations, bilinear Bäcklund transformations of a reduced equation," Applied Mathematics and Computation, vol. 219, no. 11, pp. 5837-5848, 2013.

[17] T. Xia, X. Chen, and D. Chen, "Darboux transformation and soliton-like solutions of nonlinear Schrödinger equations," Chaos, Solitons \& Fractals, vol. 26, no. 3, pp. 889-896, 2005.

[18] W. X. Ma, "Exact solutions to Tu system through Painlevé analysis," Journal of Fudan University. Natural Science, vol. 33, no. 3, pp. 319-326, 1994.

[19] E. G. Reyes, "Pseudo-spherical surfaces and integrability of evolution equations," Journal of Differential Equations, vol. 147, no. 1, pp. 195-230, 1998.

[20] G. M. Gharib, "Surfaces of a constant negative curvature," International Journal of Differential Equations, vol. 2012, Article ID 720687, 17 pages, 2012.

[21] M. J. Ablowitz and P. A. Clarkson, Solitons, Nonlinear Evolution Equations and Inverse Scattering, vol. 149, Cambridge University Press, Cambridge, UK, 1991.

[22] H. Eleuch and Y. V. Rostovtsev, "Analytical solution to sineGordon equation," Journal of Mathematical Physics, vol. 51, Article ID 093515, 2010.

[23] Y. Matsuno, "A direct method for solving the generalized sineGordon equation," Journal of Physics A: Mathematical and Theoretical, vol. 43, Article ID 105204, 2010.

[24] T. Aktosun, F. Demontis, and C. van der Mee, "Exact solutions to the sine-Gordon equation," Journal of Mathematical Physics, vol. 51, Article ID 123521, 2010.

[25] G. Hariharan, "Haar wavelet method for solving the KleinGordon and the Sine-Gordon equations," International Journal of Nonlinear Science, vol. 11, no. 2, pp. 180-189, 2011.

[26] N. Kamran and K. Tenenblat, "On differential equations describing pseudo-spherical surfaces," Journal of Differential Equations, vol. 115, no. 1, pp. 75-98, 1995.

[27] J. J. Klein, "Geometrical interpretation of the solutions of the sine-Gordon equation," Journal of Mathematical Physics, vol. 26, no. 9, pp. 2181-2185, 1985.

[28] W.-H. Steeb, Continuous Symmetries, Lie Algebras, Differential Equations and Computer Algebra, World Scientific, River Edge, NJ, USA, 1996.

[29] J. K. Perring and T. H. R. Skyrme, "A model unified field equation," Nuclear Physics B, vol. 31, pp. 550-555, 1962.

[30] A. M. Wazwaz, "Exact solutions to the double sinh-Gordon equation by the tanh method and a variable separated ODE method," Computers \& Mathematics with Applications, vol. 50, no. 10-12, pp. 1685-1696, 2005.

[31] S. Tang and W. Huang, "Bifurcations of travelling wave solutions for the generalized double sinh-Gordon equation," Applied Mathematics and Computation, vol. 189, no. 2, pp. 1774-1781, 2007. 
[32] R. K. Dodd and R. K. Bullough, "Bäcklund transformations for the sine-Gordon equations," Proceedings of the Royal Society of London A: Mathematical, Physical and Engineering Sciences, vol. 351, no. 1667, pp. 499-523, 1976.

[33] C. Rogers and W. F. Shadwick, Bäcklund Transformations and Their Applications, vol. 161, Academic Press, New York, NY, USA, 1982. 


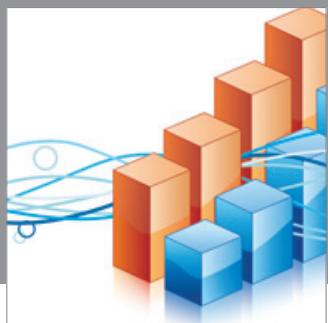

Advances in

Operations Research

mansans

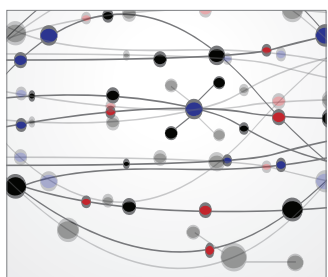

The Scientific World Journal
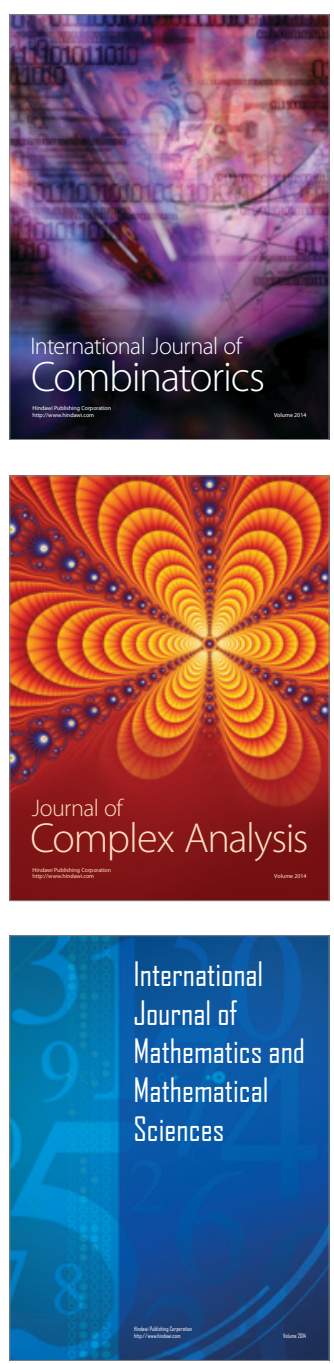
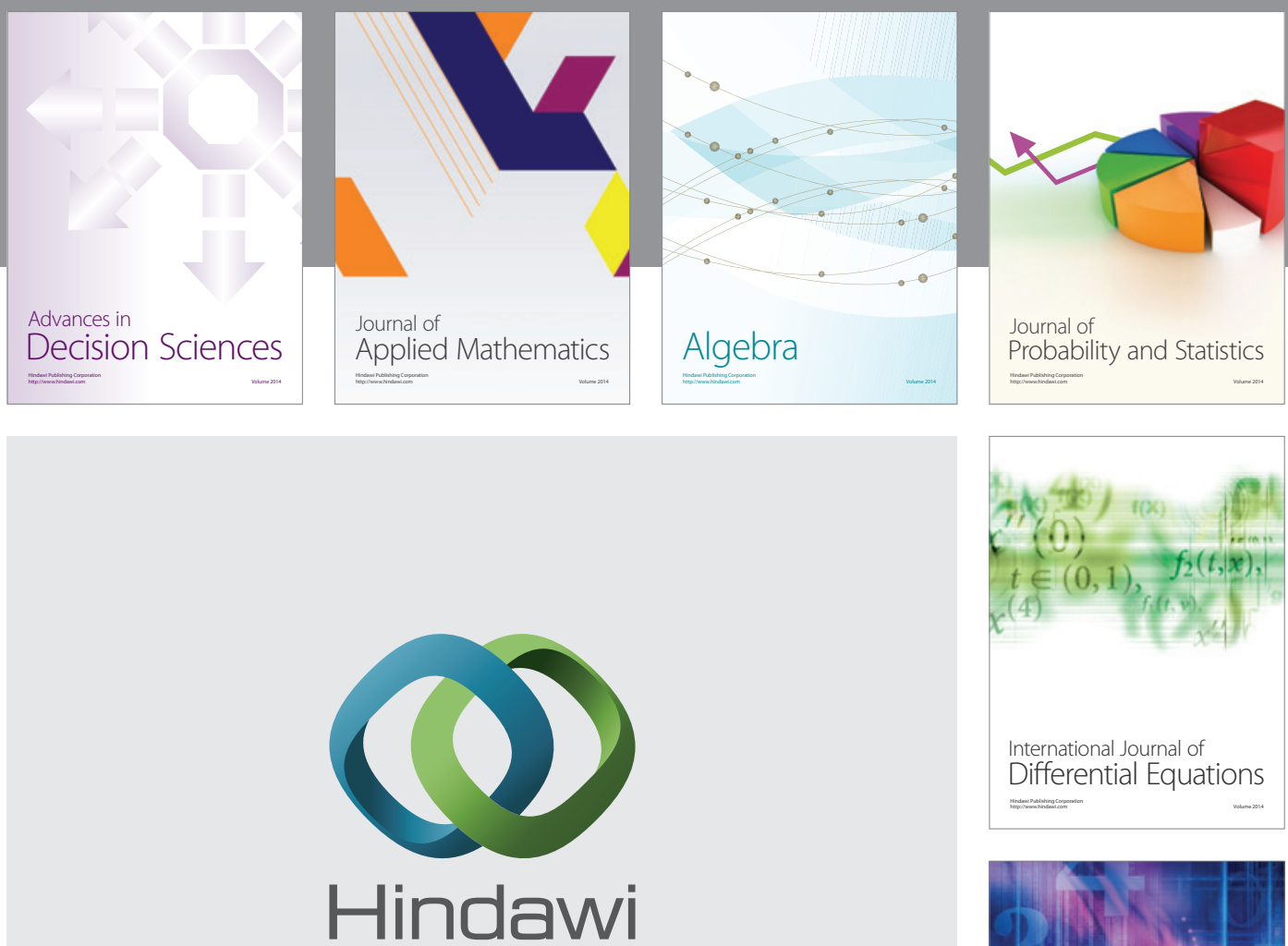

Submit your manuscripts at http://www.hindawi.com
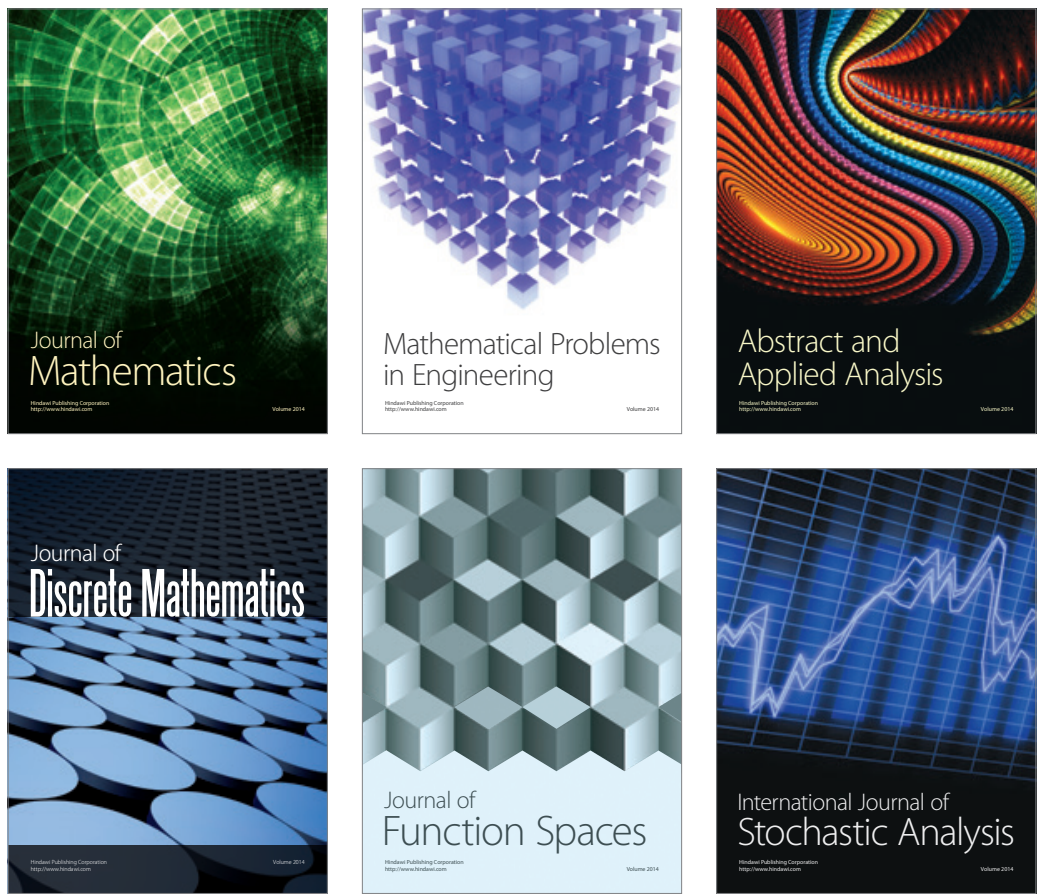

Journal of

Function Spaces

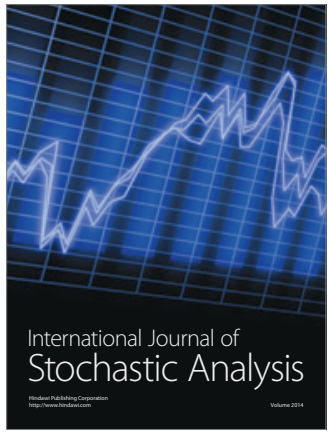

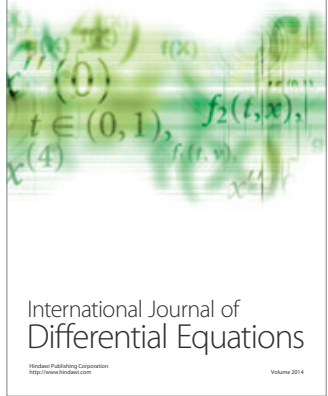
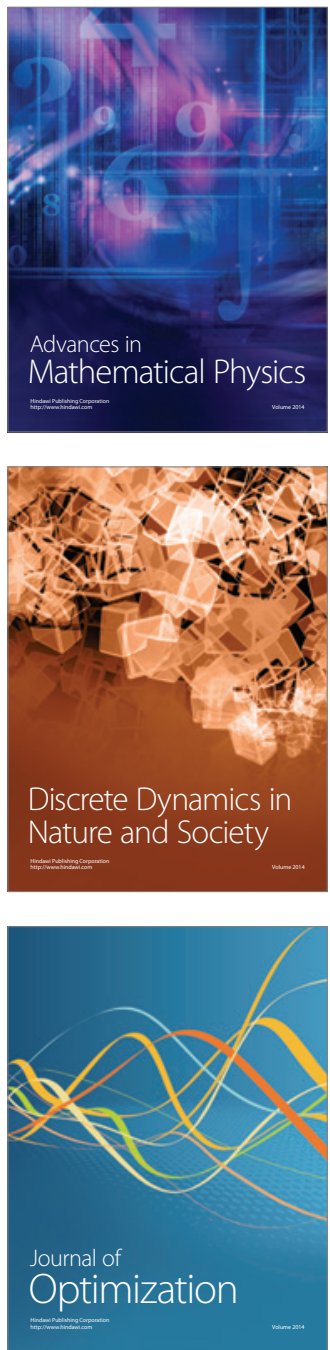